\title{
FNAL-NICADD Extruded Scintillator
}

\author{
D. Beznosko, A. Bross, A. Dyshkant, A. Pla-Dalmau V. Rykalin
}

\begin{abstract}
The possibility to produce a scintillator that satisfies the demands of physicists from different science areas has emerged with the installation of an extrusion line at Fermi National Accelerator Laboratory (FNAL). The extruder is the product of the fruitful collaboration between FNAL and Northern Illinois Center for Accelerator and Detector Development (NICADD) at Northern Illinois University (NIU). The results from the light output, light attenuation length and mechanical tolerance indicate that FNAL-NICADD scintillator is of high quality. Improvements in the extrusion die will yield better scintillator profiles and decrease the time needed for initial tuning. This paper will present the characteristics of the FNALNICADD scintillator based on the measurements performed. They include the response to MIPs from cosmic rays for individual extruded strips and irradiation studies where extruded samples were irradiated up to $1 \mathrm{Mrad}$. We will also discuss the results achieved with a new die design. The attractive perspective of using the extruded scintillator with MRS (Metal Resistive Semiconductor) photodetector readout will also be shown.
\end{abstract}

\section{INTRODUCTION}

state-of-the-art system for the production of extruded scintillator has recently been installed and commissioned at Fermi National Accelerator Laboratory (FNAL). The facility is the result of the collaboration between FNAL and Northern Illinois University's Northern Illinois Center for Accelerator and Detector Development (NICADD).

In general, the scintillator choice for a given project is based on the compromise between light output, readout system and cost. Cast plastic scintillator may cost between $\$ 40-60$ per $\mathrm{kg}$. In contrast, extruded scintillator that has significantly lower price may be used if a wavelength shifting (WLS) fiber readout system is used. With this approach, the Main Injector Neutrino Oscillation Search (MINOS) experiment was able to build two detectors that required almost 300 tons of finished extruded plastic scintillator [1]. The overall cost of the extruded plastic scintillator for MINOS was about $\$ 10$ per $\mathrm{kg}$. The ALICE (A Large Ion Collider Experiment) calorimeter upgrade at the

Manuscript received August 28, 2004. This work was supported in part by the U.S. Department of Education under Grant No. P116Z010035, the Department of Energy, and the State of Illinois Higher Education Cooperation Act. The work at FNAL was supported by the U.S. Department of Energy under contract No. DE-AC02-76CHO3000.

D. Beznosko, A. Dyshkant, V. Rykalin are with Northern Illinois University, DeKalb, IL 60115 USA (telephone: 815-753-3504, email: rykalin@fnal.gov).

A. Bross, A. Pla-Dalmau are with Fermi National Accelerator Laboratory, Batavia, IL 60510 USA (telephone: 630-840-3985, email: pla@fnal.gov).
Large Hardon Collider (LHC) needs 15 tons of scintillator. Preliminary R\&D studies [2] have investigated the possibility of using extruded scintillator. This technology can be the appropriate solution when a special scintillator shape is necessary. The emerging (stage 1 approved) MINERvA (Main Injector Experiment $\mathrm{v}$-A) [3] intends to use a triangle-shaped extruded scintillator as an active target. The triangular shape of the strips (approximately $2 \mathrm{~m}$ in length) makes the extrusion process an ideal option. The shape and the tolerance of the extrusion profile die can be optimized by means of a polymer fluid dynamics simulation process. We have made the initial attempts to develop a possible strategy for effective die design for profile extrusion. Possible applications of the extruded scintillator in new experiments will be addressed.

\section{EXTRUDER}

For our extruded scintillator, we use a continuous in-line compounding and extrusion process [4], [5]. The extrusion line is shown in Fig 1. This method allows us to minimize the handling of the polystyrene pellets and dopants (1\% (by weight) 2,5-diphenyloxazole (PPO) and $0.03 \%$ (by weight) 1,4-bis[2-(5-phenyloxazolyl)]benzene (POPOP)) that are the two major components of the extruded scintillator. A computerized system regulates the delivery of these components with high precision. During the test runs, we have been optimizing the different parameters of the extrusion process such as: correlation between speed of the puller and feed rate and temperature and pressure in the vacuum tank.

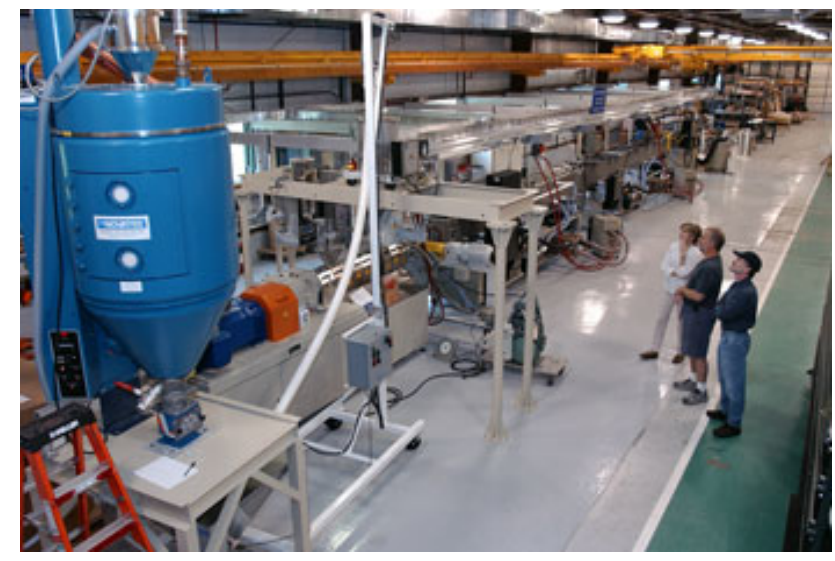

Fig. 1. Extrusion line. 
With existing dies we were able to produce scintillator strips of different shapes, some with holes for a WLS fiber and some without holes for routing the top surface as needed (Fig. 2). The presence of the extruded hole makes insertion of the fiber possible and saves additional expenses. Fig. 3 displays the scintillator strip with two extruded holes.

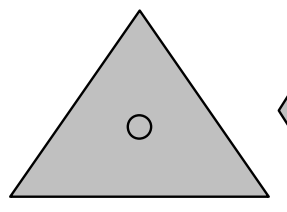

a)

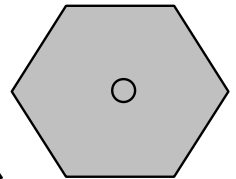

b)

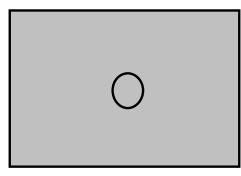

c)

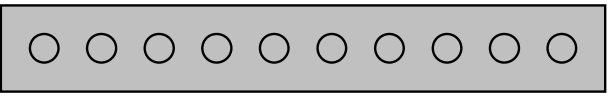

d)

Fig. 2. Different shapes of extruded scintillator, all shapes shown have the extruded hole for WLS fiber insertion. a) scintillator for MINERvA, b) scintillator for calorimeter application with pre-shower, c) shape used in R\&D studies, d) scintillator strip with up to 10 holes also used in R\&D.

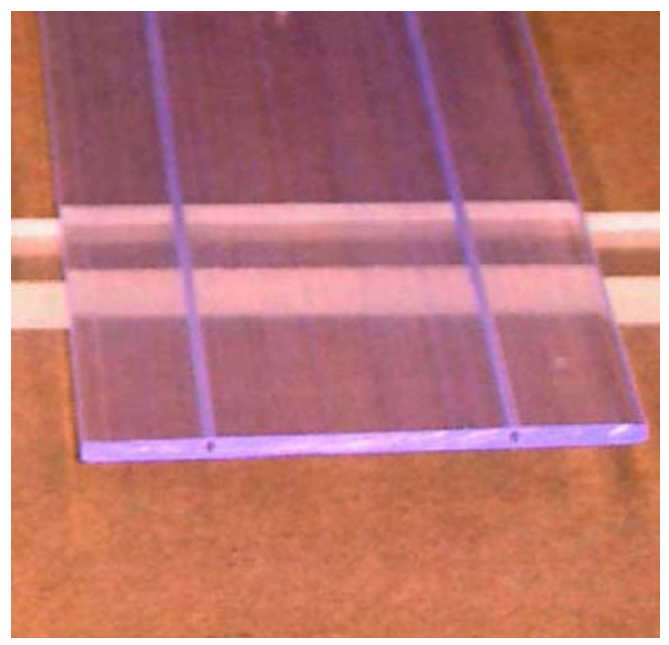

Fig. 3. Scintillator strip with two extruded holes.

The mechanical tolerances for the width and height of the extruded scintillator profiles can be closely controlled. Measurements performed on scintillator strips extruded at Fermilab (nominal size: $100 \mathrm{~mm}$ wide by $5 \mathrm{~mm}$ thick) yielded an average width of $101.33 \pm 0.17 \mathrm{~mm}$ evaluated over 300 strips ( $1 \mathrm{~m}$ long each) at 50-cm intervals (Fig. 4), and average thickness of $4.98 \pm 0.03 \mathrm{~mm}$ evaluated over 300 strips $(1 \mathrm{~m}$ long each) at 20-cm intervals (Fig. 5).

Other properties of the extruded material were studied as well, such as attenuation length, light yield, uniformity of response and radiation damage.

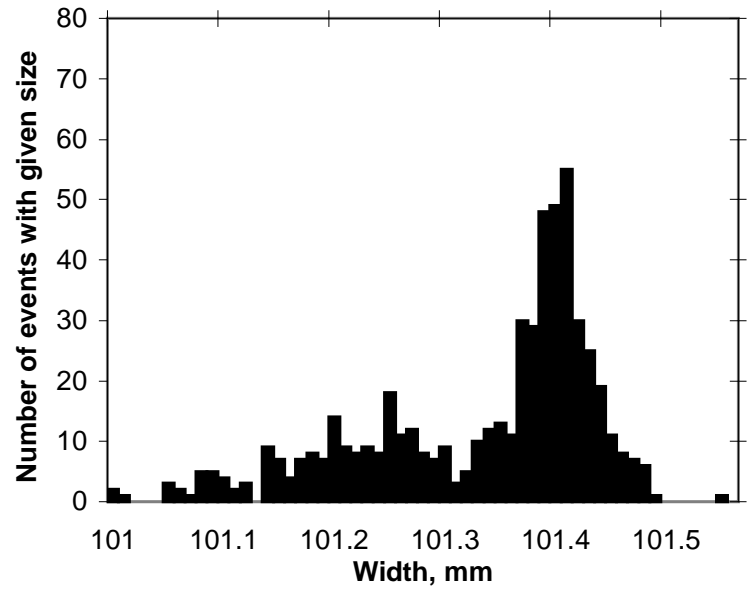

Fig. 4. Histogram of the width for extruded scintillator strips.

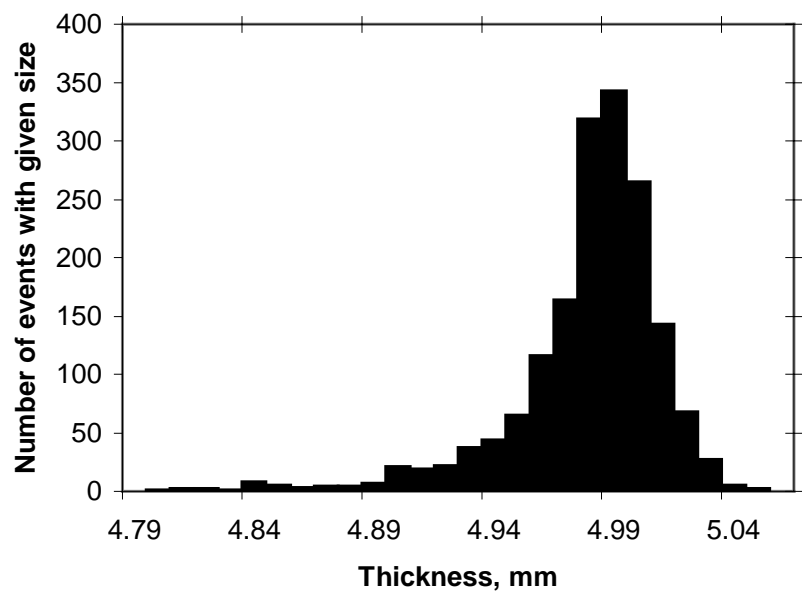

Fig. 5. Histogram of the thickness for extruded scintillator strips.

\section{A. Optical Attenuation Length}

A sophisticated drying procedure with a nitrogen purging system allows the elimination of the humidity effects and the degradation of the plastic due to the presence of oxygen. The drying system and a vacuum station at the last stage of the extruder barrel improve the final optical quality of the scintillator plastic.

The light attenuation length (LAL) was measured for the following scintillators: FNAL-NICADD and BC404 cast scintillator [6]. All pieces were cut and machined to be $2 \times 0.5 \times 100 \mathrm{~cm}^{3}$. All edges were polished to the same level. The end furthest from the PMT was painted black. The PMT (Hamamatsu R580, with a bialkali (K-Cs) photocathode) was directly coupled to the other edge of the scintillator strip. All samples were wrapped with the same Tyvek [7] sleeve. A ${ }^{137} \mathrm{Cs}$ radioactive source was placed at different distances from the PMT, with $0 \mathrm{~cm}$ being the point furthest away from the PMT and $100 \mathrm{~cm}$ being the point closest to the PMT. The value of 
the PMT anode current was read out by the picoammeter and was plotted against distance in Fig. 6.

The value of the exponent of the fit parameter corresponds to the inverse of LAL for each scintillator sample tested, expressed in $\mathrm{cm}$. The cast scintillator sample (BC404) exhibits the higher value for LAL than FNAL-NICADD sample, as expected. The actual value of LAL is dependent on the geometry of the measured sample and particulars of the setup and method used, thus absolute values are not as important as the comparison between the values.

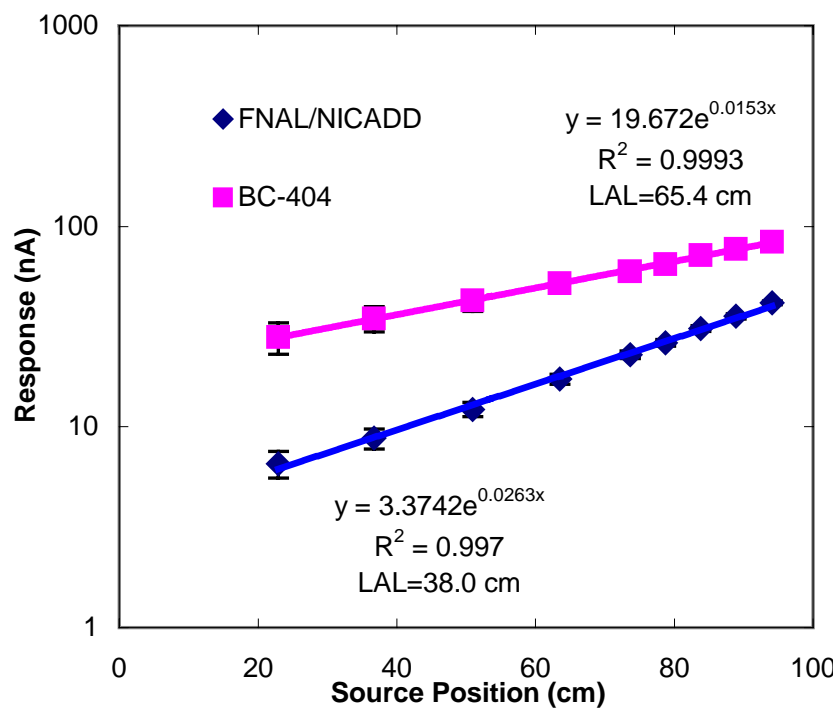

Fig. 6. Attenuation length for two different scintillators. The point closest to the PMT is at $100 \mathrm{~cm}$.

\section{B. Light Output}

The extruded scintillator is composed of polystyrene pellets (Dow Styron $663 \mathrm{~W}$ ), 1\% PPO and $0.03 \%$ POPOP. To compare the light output level of extruded scintillator with other brand scintillators, the light output was measured for the following scintillators: FNAL-NICADD extruded, BC408 [6] and Kuraray [8] SCSN-81. In this measurement, small pieces $\left(2 \times 0.5 \times 2 \mathrm{~cm}^{3}\right)$ of scintillator, wrapped in the same material, were measured by a PMT (Hamamatsu R580) directly, which was read out by a picoammeter. $\mathrm{A}{ }^{106} \mathrm{Ru}$ radioactive source was used and always placed at the same distance from the PMT. The results are shown in Table I. The response is normalized to $1 \mathrm{~mm}$ thickness. The results are not corrected for spectral differences in the light emission and PMT response. This is the light output of the bulk scintillator. In small pieces the optical attenuation length differences among cast and extruded samples do not play a role. The FNAL-NICADD extruded scintillator is a polystyrene-based scintillator (such as SCSN-81) with an intrinsic $20 \%$ drop in scintillation yield when compared to poly(vinyltoluene)-based scintillator (such as BC408).
TABLE I SCINTILLATOR RESPONSE

\begin{tabular}{cc}
\hline Type & Response $(\mu \mathrm{A})$ \\
\hline FNAL-NICADD & $2.0 \pm 0.2$ \\
BC408 & $2.7 \pm 0.2$ \\
KURARAY SCSN-81 & $2.0 \pm 0.2$ \\
\hline
\end{tabular}

In order to measure the light output of an extruded scintillator with a WLS fiber using cosmic ray muons, we used the following setup. A FNAL-NICADD scintillator strip $\left(10 \times 0.5 \times 100 \mathrm{~cm}^{3}\right)$ was wrapped in Tyvek. A WLS fiber (Kuraray Y-11) was inserted in the hole. A Metal-ResistorSemiconductor (MRS) [9], [10] silicon sensor with $1-\mathrm{mm}^{2}$ photosensitive area was used for the readout. With a $1.2-\mathrm{mm}$ diameter fiber, we measured $\sim 17$ photo electrons (PE) with this sensor that has quantum efficiency at $500 \mathrm{~nm}$ of $\sim 25 \%$ [9]. A MINOS extruded scintillator sample was measured for comparison using the same 1.2-mm diameter WLS fiber. However, the MINOS sample had a co-extruded reflective coating. In addition, measurements using the FNAL-NICADD extruded scintillator strips with $1.0-\mathrm{mm}$ and $1.5-\mathrm{mm}$ diameter fibers were carried out. The increase in response is not proportional to the increase in the area of the WLS fiber because the MRS sensor is only $1 \mathrm{~mm}^{2}$.

Table II summarizes the results of measurements done using the 5-mm thick extruded scintillator and MRS sensor with Kuraray Y-11 WLS fibers of different diameters. Here the response in PE refers to each photon detected by MRS (i.e. the total number of incident photons on the sensor is approximately the response in PE times 4). For sensor calibration and example of the signal spectra, see Appendix.

TABLE II

FIBER THICKNESS AND RESPONSE

\begin{tabular}{lll}
\hline $\begin{array}{l}\text { FIBER } \\
\text { DIAMETER } \\
(\mathrm{MM})\end{array}$ & $\begin{array}{l}\text { SCINTILLATOR TYPE } \\
\text { AND THICKNESS }\end{array}$ & $\begin{array}{l}\text { AVERAGE } \\
\text { RESPONSE } \\
(\mathrm{PE})\end{array}$ \\
\hline 1.0 & $\begin{array}{l}\text { FNAL-NICADD } \\
\text { Extruded, 5mm }\end{array}$ & 14.5 \\
1.2 & $\begin{array}{l}\text { FNAL-NICADD } \\
\text { Extruded, 5mm }\end{array}$ & 17 \\
1.5 & $\begin{array}{l}\text { FNAL-NICADD } \\
\text { Extruded, 5mm }\end{array}$ & 20.5 \\
1.2 & MINOS Extruded, 10mm & 22.1
\end{tabular}




\section{Uniformity of Response}

Uniformity of the response of the extruded scintillator was measured across the $10 \times 0.5 \times 100 \mathrm{~cm}^{3}$ strip and a hexagonal cell, with area of $9 \mathrm{~cm}^{2}$ and $5 \mathrm{~mm}$ thick. The non-uniformity of response across a scintillator strip was measured and yielded a value of $\sim 4 \%$. The result of a similar non-uniformity scan for the hexagonal cell is $<3 \%$.

The results of these measurements are presented in Fig. 7 for the scan across the scintillating strip and in Fig. 8 for the scan across the hexagonal cell.

In Fig. 7, the structure shows that response is somewhat larger near the WLS fibers, but smaller when the source is directly above and there is not as much scintillating material to produce light. The error bars are not shown because they would be on the same scale that the markers used and would not be visible.

In Fig. 8, the same increase is observed in the fiber region. However, because it is a groove and not a co-extruded hole, there is still enough material between the fiber and the source not to produce a slight dip in response as in the same of the extruded strips. Again, the error bars are not shown because they would be on the same scale that the markers used.

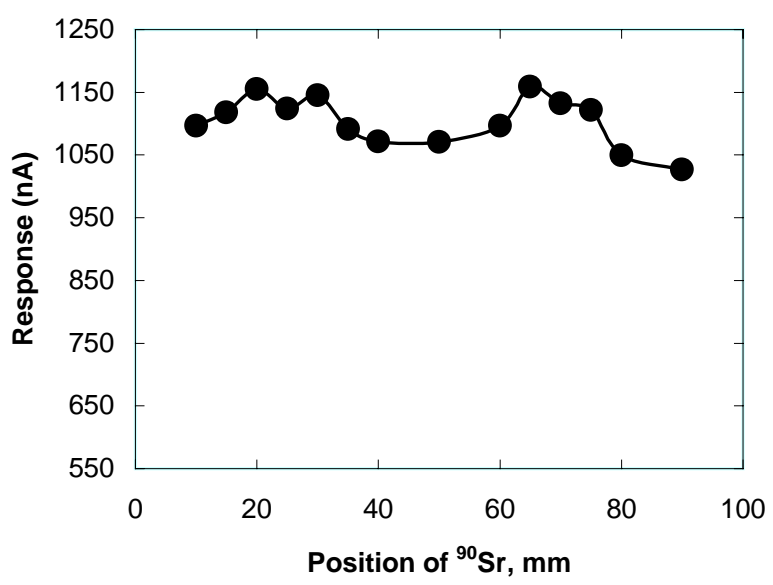

Fig. 7. Non-uniformity of response across the scintillator strip with two holes.

\section{Radiation Damage}

Several samples $\left(2 \times 0.5 \times 2 \mathrm{~cm}^{3}\right)$ prepared from extruded FNAL-NICADD scintillator strips were irradiated to 0.5 and 1 Mrad in air utilizing a ${ }^{60} \mathrm{Co}$ gamma source with a dose rate of $0.8 \mathrm{Mrad}$ per hour. Pulse height $(\mathrm{PH})$ measurements on these samples were performed using $1 \mathrm{MeV}$ conversion electrons from a ${ }^{207} \mathrm{Bi}$ radioactive source before and 85 days after the irradiation. The results are shown in Table III. PH is shown in arbitrary units. The overall reproducibility of these measurements is dominated by the preparation and condition of the sample surface and is approximately $\pm 5 \%$.

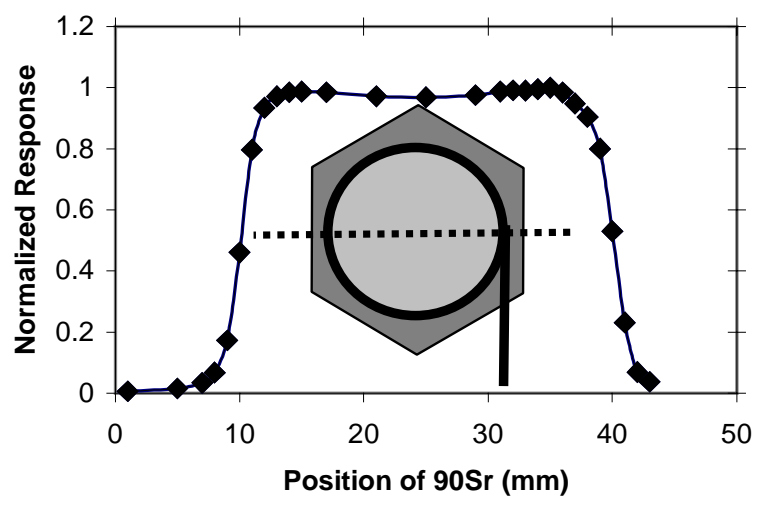

Fig. 8. Non-uniformity of response across the hexagonal cell. Response is normalized to the maximum value. The schematics of cell indicates the direction of scan.

Therefore we conclude that there is no radiation damage detectable up to $1 \mathrm{Mrad}$ as previously observed with cast scintillator samples. The delay in measuring the samples after irradiation is to allow for the natural annealing to occur. Measurements of light yield right after the irradiation were not carried over for technical reasons. Although the samples were irradiated in air, they underwent annealing because the dose rate had been high enough that the oxygen diffusion rate was smaller than its consumption rate. The final results are similar to those of an irradiation under inert conditions with the exception of the formation of oxidation products on the surface of the samples.

In addition, the transmittance of the scintillator was measured, both before and after the irradiation, and after 85day annealing as well (Fig. 9). As with the light yield, no significant changes were detectable. The minor variations among the spectra in Fig. 9 are within the accuracy of the instrument. The measurements were referenced to air as indicated by the solid absorption at low wavelengths and the poor transmission at long wavelengths. Since no significant change in transmittance was observed, a decrease of the light yield of the scintillator itself is most likely responsible for the changes seen in Table III.

TABLE III

Pulse HeIGHT BEFore AND AFTER IRRAdiation

\begin{tabular}{llll}
\hline Dose (Mrad) & $\begin{array}{l}\text { Before } \\
\text { Irradiation }\end{array}$ & $\begin{array}{l}\text { After } \\
\text { Irradiation }\end{array}$ & $\begin{array}{l}\text { Light yield } \\
\text { loss }(\%)\end{array}$ \\
\hline 0.5 & $266 \pm 7$ & $264 \pm 8$ & none \\
1 & $273 \pm 6$ & $261 \pm 7$ & $\sim 5$ \\
\hline
\end{tabular}




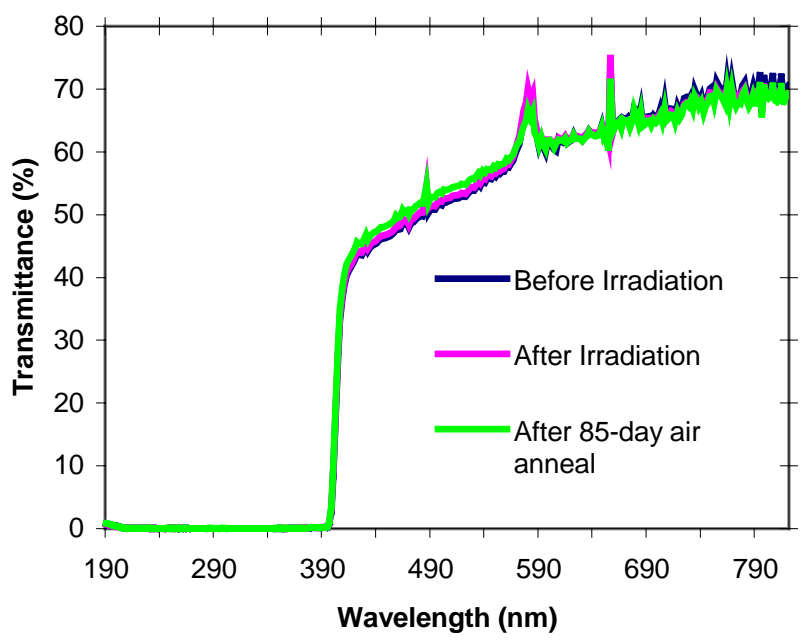

Fig. 9. Transmittance of the extruded scintillator before, after and 85 days after irradiation.

\section{CONCLUSIONS}

The first R\&D runs on the new extrusion line at FNAL have shown the attractiveness of the FNAL-NICADD scintillator for different applications in Nuclear and High Energy Physics. The mechanical tolerances are $\pm 0.6 \%$ for thickness and $\pm 0.16 \%$ for width. The scintillator response, or Light Yield (LY), is within $74 \%$ of BC408 and is equal to Kuraray SCSN-81. The LY non-uniformity is $<3 \%$ for the small hexagonal cell and $\sim 4 \%$ for the long strips. No radiation damage is detected for doses up to $1 \mathrm{Mrad}$ in air at high dose rate.

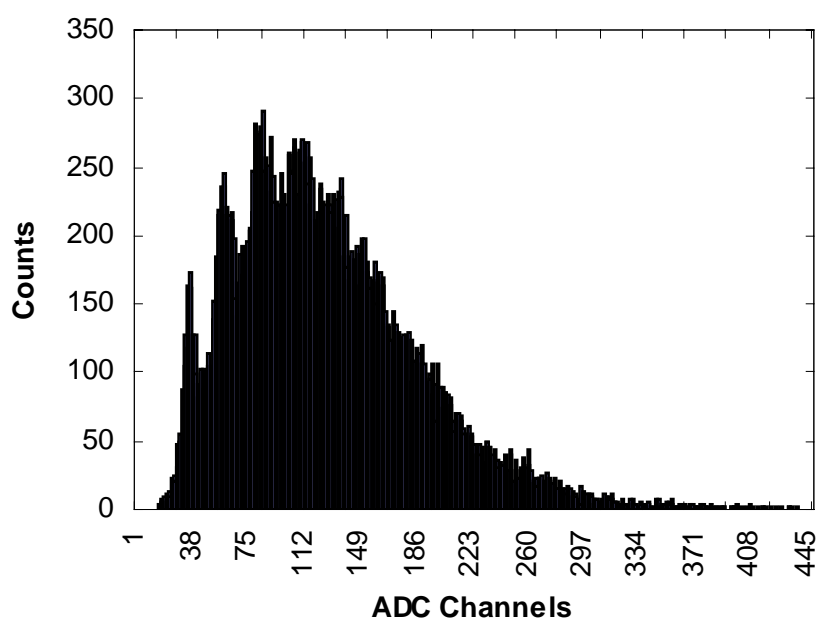

Fig. 10. MRS calibration data at $50 \mathrm{~V}$ using LED as light source. 1PE here $\sim 23$ ADC Channels. Pedestal in channel 38.

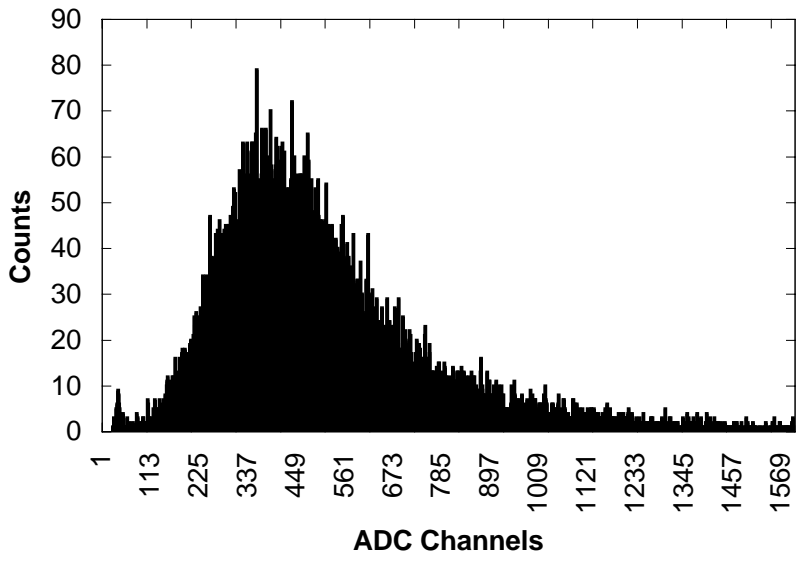

Fig. 10. MRS spectra for $1.2 \mathrm{~mm}$ Y11 fiber and $5 \mathrm{~mm}$ thick extruded scintillator, for cosmic ray muons. Using calibration above, get $\sim 17.2$ PE. Pedestal in channel 38 .

\section{APPENDIX}

The calibration data and the spectra obtained for cosmic ray muons using MRS sensor and $1.2 \mathrm{~mm}$ diameter Y11 fiber are provided in Fig.10 and Fig. 11 respectively.

\section{ACKNOWLEDGMENT}

We thank Brian Wood (UIC) and Elena Baldina (FNAL) for their valuable help with measurements.

\section{REFERENCES}

[1] A. Pla-Dalmau et al, "Extruded Plastic Scintillator for the MINOS Calorimeters", Frascati Physics Series XXI (2000), 513-522.

[2] O.A. Grachov, T.M. Cormier, A. Pla-Dalmau, A. Bross, V. Rykalin, "A Study Of New FNAL-NICADD Extruded Scintillator As Active Media Of Large EMCAL Of ALICE At LHC”, FNAL-CONF-04-046, XI int. Conf. Calor 2004, Italy, 2004

[3] D. Drakoulakos et al. [the MINERvA collaboration], "Proposal to Perform a High-Statistics Neutrino Scattering Experiment Using a Finegrained Detector in the NuMI Beam". http://www.pas.rochester.edu/minerva/

[4] A. Pla-Dalmau, A. Bross, V. Rykalin, "Extruding Plastic Scintillator at Fermilab", IEEE Nuclear Science Symposium Conference Record Portland, OR, Oct. 19-25 2003 (FERMILAB-Conf-03-318-E).

[5] A. Pla-Dalmau, A.D. Bross and K.L. Mellott, "Low-Cost Extruded Plastic Scintillator", Nucl. Instr. Meth. A466 (2001), 482-491.

[6] SAINT-GOBAIN (Bicron), 12345 Kinsman Road, Newbury, OH 44065, USA.

[7] A. Dyshkant, D. Beznosko, G. Blazey, D. Chakraborty, K. Francis, D. Kubik et al., "Small scintillating cells as the active elements in a digital hadron calorimeter for the e+e- linear collider detector" 2004 J. Phys. G: Nucl. Part. Phys. 30 N1-N16

[8] Kuraray America Inc., 200 Park Ave, NY 10166,USA; 3-1-6, NIHONBASHI, CHUO-KU, TOKYO 103-8254, JAPAN.

[9] V. Golovin et al., "Limited Geiger-Mode Silicone Photodode With Very High Gain:, Nucl.Phys.Proc.Suppl. 61B: 347-352, 1998.

[10] D. Beznosko, G. Blazey, D. Chakraborty, A. Dyshkant, K. Francis, D. Kubik et al., "Investigation of a Solid State Photodetector", NIM A, Volume 545, Issue 3, 21 June 2005, Pages 727-737. 